\section{BMJ Open Respiratory Research}

\title{
Automatic versus manual oxygen titration using a novel nasal high-flow device in medical inpatients with an acute illness: a randomised controlled trial
}

\author{
James Harper (D) , ,2,3 Nethmi Kearns, ${ }^{1,3}$ Grace Bird, ${ }^{1,2,3}$ Irene Braithwaite (D) , \\ Allie Eathorne, ${ }^{1}$ Nicholas Shortt, ${ }^{1}$ Mark Weatherall, ${ }^{4}$ Richard Beasley ${ }^{1,2,3}$
}

To cite: Harper J, Kearns N, Bird G, et al. Automatic versus manual oxygen titration using a novel nasal high-flow device in medical inpatients with an acute illness: a randomised controlled trial. BMJ Open Resp Res 2021;8:e000843. doi:10.1136/ bmjresp-2020-000843

- Additional online supplemental material is published online only. To view, please visit the journal online (http://dx.doi.org/10. 1136/bmjresp-2020-000843).

Received 20 November 2020 Accepted 11 July 2021

Check for updates

(c) Author(s) (or their employer(s)) 2021. Re-use permitted under CC BY-NC. No commercial re-use. See rights and permissions. Published by BMJ.

${ }^{1}$ Medical Research Institute of New Zealand, Wellington, New Zealand

${ }^{2}$ Victoria University of Wellington, Wellington, New Zealand

${ }^{3}$ Capital and Coast District Health Board, Wellington, New Zealand

${ }^{4}$ University of Otago Wellington, Wellington, New Zealand

Correspondence to Dr James Harper; james.harper@mrinz.ac.nz

\section{ABSTRACT}

Background Guideline recommendations state oxygen should be administered to acutely unwell patients to achieve a target oxygen saturation $\left(\mathrm{SpO}_{2}\right)$ range. The current practice of manual oxygen titration frequently results in $\mathrm{SpO}_{2}$ outside of a prescribed range. The aim of this study was to assess the efficacy of automatic oxygen titration using a closed-loop feedback system to achieve $\mathrm{SpO}_{2}$ within a prescribed target range

Methods An open-label randomised parallel group trial was undertaken comparing automatic oxygen titration using a novel nasal high-flow device to manual oxygen titration using nasal high flow. Medical inpatients requiring oxygen therapy in Wellington Regional Hospital, New Zealand with a prescribed target $\mathrm{SpO}_{2}$ range of $88 \%-92 \%$ or $92 \%-96 \%$ were recruited and randomised equally between the interventions for a period of 24 hours. The primary outcome was the proportion of time spent with $\mathrm{SpO}_{2}$ within the prescribed range.

Results 20 patients were included in the analysis. Automatic oxygen titration resulted in a median (IQR) $96.2 \%$ (95.2-97.8) of time within the target range compared with $71 \%(59.4-88.3)$ with manual titration; difference $(95 \% \mathrm{Cl}) 24.2 \%$ (7.9\% to $35 \%), p<0.001$. There was a reduction in the time spent with $\mathrm{SpO}_{2} \geq 2 \%$ above and $\geq 2 \%$ below range in the automatic titration group, although the point estimate for the differences were small; $-1 \%(-8.2 \%$ to $-0.04 \%), p=0.017$ and $-2.4 \%(-11.5 \%$ to $0.3 \%), p=0.05$ respectively.

Conclusions Nasal high-flow with automatic oxygen titration resulted in a greater proportion of time spent with $\mathrm{SpO}_{2}$ in target range compared with manual titration. Trial registration The trial was registered with the Australian and New Zealand Clinical Trials Registry (ACTRN12619000901101).

\section{INTRODUCTION}

Oxygen is commonly administered in hospital with the aim of treating hypoxaemia. ${ }^{1}$ International guidelines recommend titration of oxygen in order to achieve a target peripheral oxygen saturation $\left(\mathrm{SpO}_{2}\right)$ range in acutely

\section{Key messages}

Does nasal high-flow with automatic oxygen titration result in a greater proportion of time spent with oxygen saturation $\left(\mathrm{SpO}_{2}\right)$ within a prescribed target range compared to nasal high-flow with the standard practice of manual oxygen titration in medical inpatients?

- In this randomised controlled trial, automatic oxygen titration resulted in a significantly greater proportion of time spent with $\mathrm{SpO}_{2}$ within target range.

- This is the first study to demonstrate that automatic oxygen titration using nasal high flow is superior to the current practice of manual oxygen titration based upon intermittent $\mathrm{SpO}_{2}$ measurements in medical inpatients with an acute illness, reducing the potential risk of over and under oxygenation.

unwell patients. ${ }^{23}$ Deviation from this target $\mathrm{SpO}_{2}$ range results in progressive risk of harm $^{4}$; an $\mathrm{SpO}_{2}$ below $90 \%$ in a hospital ward setting is associated with a 2.4-fold increase in risk of in-hospital mortality ${ }^{5}$ and the liberal administration of oxygen is associated with a $1 \%$ increase in risk of mortality per $1 \%$ increase in $\mathrm{SpO}_{2}$ above $96 \%,{ }^{6} 7$ although recent studies have suggested equipoise. ${ }^{8} 9$ A target $\mathrm{SpO}_{2}$ range of $92 \%-96 \%$ is recommended by the Thoracic Society of Australia and New Zealand (TSANZ) ${ }^{3}$ and a range of $94 \%-98 \%$ by the British Thoracic Society. ${ }^{2}$ Both guidelines recommend a lower target range of $88 \%-92 \%$ in patients who are at risk of hypercapnic respiratory failure. ${ }^{23}$

In current clinical practice, manual oxygen titration based on intermittent $\mathrm{SpO}_{2}$ measurement is used in order to achieve a prescribed target $\mathrm{SpO}_{2}$ range. This process has remained unchanged for several decades, despite evidence that patients receiving oxygen 
frequently have an $\mathrm{SpO}_{2}$ outside this range. ${ }^{10}$ Recently, oxygen delivery systems using a closed-loop feedback system have been developed which enable continuous automated adjustment of a delivered oxygen concentration. ${ }^{11-15}$ Studies of these devices have demonstrated patients spend a greater proportion of time with $\mathrm{SpO}_{2}$ within target range compared with the standard practice of manual oxygen titration both in the emergency department (ED), ${ }^{12}$ ward setting ${ }^{13}$ and following abdominal and thoracic surgery. ${ }^{11}$

To date, studies of automated oxygen titration in adults have involved conventional low-flow oxygen. Nasal high flow (NHF) involves the delivery of heated, humidified gas at high flow via a wide bore nasal cannula. This method of oxygen delivery has a number of beneficial physiological effects ${ }^{16-20}$ and is associated with a reduced risk of endotracheal intubation when compared with conventional oxygen therapy in patients with acute hypoxaemic respiratory failure. ${ }^{21} 22$ As a result, NHF has become an established therapy for selected patients with hypoxaemia in the acute care setting. ${ }^{23}$

The Airvo $3^{\mathrm{TM}}$ device (Fisher and Paykel Healthcare, Auckland, New Zealand) is a novel NHF system which is able to automatically titrate the delivered oxygen concentration in order to achieve a user set target $\mathrm{SpO}_{2}$ using a closed-loop feedback mechanism. This is achieved by automatic adjustment of the oxygen concentration using an intelligent algorithm that adapts output based on $\mathrm{SpO}_{2}$ responsiveness. A prototype version of this device has demonstrated responsiveness to changes in $\mathrm{SpO}_{2}$ outside a target range in a laboratory-based exercise test. ${ }^{24}$ This is the first study to investigate the use of NHF with automatic oxygen titration in adults with an acute illness. This aim of this study was to compare NHF with automatic oxygen titration to NHF with manual oxygen titration in patients who had been admitted to hospital with an acute medical illness. Our hypothesis was that NHF with automatic oxygen titration would result in a greater proportion of time spent with $\mathrm{SpO}_{2}$ within target range.

\section{METHODS}

\section{Study design}

This was an open-label randomised parallel group trial. The trial was run in accordance with Good Clinical Practice guidelines and the Declaration of Helsinki. Patients. Members of the public were not involved with the design or conduct of this study.

\section{Participants}

Patients under the care of a medical team were eligible for inclusion if they had a clinician prescribed $\mathrm{SpO}_{2}$ target range of $88 \%-92 \%$ or $92 \%-96 \%$, as per TSANZ guidelines. $^{3}$

\section{Inclusion criteria: target $\mathrm{SpO}_{2} 92 \%-96 \%$}

- Current inpatient under the care of a medical or surgical team.
- Expected duration of admission >24 hours from enrolment.

- NHF therapy already commenced or treating physician decision to start NHF or requirement for $\geq 2 \mathrm{~L} /$ min low-flow oxygen.

Inclusion criteria: target $\mathrm{SpO}_{2} 88 \%-92 \%$

- Current inpatient under the care of a medical or surgical team.

- Expected duration of admission >24 hours from enrolment.

- NHF therapy already commenced or treating physician decision to start NHF or requirement for $\geq 1 \mathrm{~L} /$ min low-flow oxygen.

\section{Common exclusion criteria}

- Age $<18$.

- Unable to tolerate a brief interruption in oxygen or high-flow therapy.

- Domiciliary use of CPAP or NIV.

- Diagnosis of obstructive sleep apnoea.

- Evidence of respiratory infection or colonisation with multidrug resistant bacteria, Pseudomonas species, Burkholderia Cepacia or mycobacteria.

- Suspected or proven infection with SARS-CoV-2 (COVID-19).

- Haemodynamic instability (systolic blood pressure $<90 \mathrm{~mm} \mathrm{Hg}$ or requirement for vasopressor or inotropic support).

- Patient receiving end of life care.

- Risk of barotrauma, as assessed by the investigator.

- Nasal or facial conditions precluding use of NHF.

- Intracranial trauma or trans-nasal neurosurgery (within 6 weeks).

- Any condition which limits the feasibility of continuous $\mathrm{SpO}_{2}$ monitoring using a finger probe such as anatomical deformity or vascular compromise.

- Pregnancy or breast feeding.

- Cognitive impairment or impaired consciousness precluding informed consent.

- Implanted electronic medical device (including insulin pump, pacemaker, neurostimulator and implantable cardioverter-defibrilator).

- Any other condition which, at the investigator's discretion, is believed may present a safety risk or impact the feasibility of the study or the study results.

\section{Exclusion criteria: target $\mathrm{SpO}_{2} 92 \%-96 \%$}

- Requirement for $>40 \%$ oxygen at time of enrolment (equivalent to $5 \mathrm{~L} / \mathrm{min}$ low-flow oxygen).

- Hypercapnia $\left(\mathrm{PCO}_{2}>45 \mathrm{~mm} \mathrm{Hg}\right)$ with or without acidosis.

- Presence of any risk factor for hypercapnic respiratory failure including: chronic obstructive pulmonary disease (COPD), cystic fibrosis, bronchiectasis, chest wall deformity or neuromuscular disease and 
investigator considers $92 \%-96 \%$ to be an inappropriate $\mathrm{SpO}_{2}$ target.

\section{Exclusion criteria: target $\mathrm{SpO}_{2} 88 \%-92 \%$}

- Requirement for $>35 \%$ oxygen at time of enrolment (equivalent to $4 \mathrm{~L} / \mathrm{min}$ low-flow oxygen)

- Respiratory acidosis $\left(\mathrm{pH}<7.35\right.$ and $\mathrm{PCO}_{2}>45 \mathrm{~mm}$ $\mathrm{Hg}$ ).

Patients with compensated hypercapnia and a target $\mathrm{SpO}_{2}$ range of $88 \%-92 \%$ could be included, however, those with an associated respiratory acidosis were excluded. The study protocol was amended to facilitate the inclusion of surgical patients due to difficulty with recruitment during the COVID-19 pandemic, however, this was ultimately not required and only medical patients were recruited. As the first study of NHF with automatic oxygen titration in the acute setting, eligibility criteria were designed to provide a study population representative of those who may receive NHF in clinical practice, while excluding those at greatest risk and those who require close monitoring in an intensive care setting.

\section{Procedures}

Patients under the care of a medical team who were receiving oxygen were identified by a study investigator and potentially suitable patients were provided with an information sheet and written informed consent was obtained. Baseline physical observations were recorded and a capillary blood gas was taken from the earlobe. Eligible participants were randomised 1:1 to NHF with automatic oxygen titration using the Airvo 3 device or NHF with manual oxygen titration using the Airvo 2 device (Fisher and Paykel Healthcare, Auckland, New Zealand) for a period of 24 hours. The Airvo 2 device is an established NHF device and is used as part of routine practice in Wellington Hospital.

The randomisation schedule was computer generated by the study statistician and incorporated into the Research Electronic Data Capture (REDCap) ${ }^{25} 26$ study electronic database. The randomisation schedule was concealed from study investigators within the REDCap database. The treatment to which each participant was randomised was automatically displayed for each recruited participant at the point of randomisation. This was an open-label study in which both participants and investigators were aware of the treatment, blinding to treatment allocation was not possible due to the nature of the intervention.

An AirSpiral heated breathing tube (Fisher \& Paykel Healthcare, Auckland, New Zealand) and Optiflow + nasal cannula (Fisher \& Paykel Healthcare, Auckland, New Zealand) was used in both groups. A laminated guide was attached to the NHF device in both groups specifying the prescribed target $\mathrm{SpO}_{2}$ range and prompting intervention if $\mathrm{SpO}_{2}$ was above or below range.

For both groups, at the start of the intervention period, a study investigator titrated the delivered oxygen concentration to achieve $\mathrm{SpO}_{2}$ as close to the mid-point of the target range as possible. The initial flow was set to $35 \mathrm{~L} / \mathrm{min}$, or the same flow as used prior to randomisation if the participant was already receiving NHF. All other clinical care continued as usual and investigators had no input into patient management during the intervention period.

\section{Automatic titration}

In the automatic titration group, an adhesive finger sensor provided feedback to the NHF device and an additional sensor was placed on the contralateral hand which was connected to an independent pulse oximeter (sat 801+ Bitmos Düsseldorf, Germany). The Airvo 3 device screen displayed $\mathrm{SpO}_{2}$, flow, temperature, $\mathrm{FiO}_{2}$ and $\mathrm{FiO}_{2}$ upper and lower limits. A logging box was connected to the Airvo 3 device which recorded data. The independent pulse oximeter was used as a safety measure. The device was able to automatically titrate the delivered oxygen concentration within a range of $10 \%$, for example, between $25 \%$ and $35 \%$. An alarm sounded on the device if the $\mathrm{SpO}_{2}$ was below range while $\mathrm{FiO}_{2}$ was at the upper limit, or if $\mathrm{SpO}_{2}$ was above range at the lower $\mathrm{FiO}_{2}$ limit, at which point nursing staff were able to increase the upper limit by $5 \%$ or reduce the lower limit by $5 \%$ as needed. High and low $\mathrm{SpO}_{2}$ alarms $(98 \%$ and $90 \%$, respectively, with target range $92 \%-96 \%$ and $94 \%$ and $86 \%$, respectively, with target range $88 \%-92 \%$ ) were also set on the NHF device and independent pulse oximeter.

\section{Manual titration}

In the manual titration group, an adhesive finger sensor was connected to a data logging box which did not display $\mathrm{SpO}_{2}$ or any other metrics. The Airvo 2 device screen displayed flow, $\mathrm{FiO}_{2}$ and temperature. Nursing staff were instructed to measure $\mathrm{SpO}_{2}$ according to usual practice; continuous oximetry was used at the discretion of the medical and nursing teams.

In both groups heart rate, $\mathrm{SpO}_{2}, \mathrm{FiO}_{2}$ and flow data were recorded by the data logging boxes every second, resulting in approximately 86400 measurements per participant. At the end of the 24-hour study period, a second capillary blood gas was taken from the earlobe, the investigational NHF device was removed and data was downloaded and processed. In the manual titration group $\mathrm{FiO}_{2}$ data analysis was assisted by visual inspection of the $\mathrm{FiO}_{2}$-time plots due to noise in the Airvo $2 \mathrm{FiO}_{2}$ data. Participants were withdrawn from the study in event of a sustained requirement for $>50 \%$ oxygen (92\%-96\% group), $>40 \%$ oxygen ( $88 \%-92 \%$ group) or an increase of more than $10 \%$ from the $\mathrm{FiO}_{2}$ at the start of the intervention period. Further withdrawal criteria are shown in the supplementary material.

An independent data safety monitoring committee (DSMC) reviewed the safety of the study after the first, second and third enrolled participants and every 10 participants thereafter. All adverse events were also 
reviewed by the DSMC. The study funder had no involvement with the DSMC.

\section{Outcomes}

The primary outcome was the proportion of time spent with $\mathrm{SpO}_{2}$ within the prescribed target range. Data with adequate signal quality (defined as $>0.5$ on a scale from zero to one) were used for the primary outcome in order to reduce the likelihood of including spurious $\mathrm{SpO}_{2}$ values resulting from motion artefact. Secondary outcomes included the proportion of time spent with $\mathrm{SpO}_{2}$ within range irrespective of signal quality and the proportion of time spent $\geq 2 \%, \geq 4 \%$ and $\geq 8 \%$ below range, as well as $\geq 2 \%$ and $\geq 4 \%$ above range. Additional secondary outcomes included heart rate, number of $\mathrm{FiO}_{2}$ (manual titration) or $\mathrm{FiO}_{2}$ range (automatic titration) adjustments, $\mathrm{FiO}_{2}$ and capillary blood gas measures. Tertiary outcomes included the proportion of time spent with $\mathrm{SpO}_{2}$ above range when the $\mathrm{FiO}_{2}$ was at the lower limit and time spent with $\mathrm{SpO}_{2}$ below range when the $\mathrm{FiO}_{2}$ was at the upper limit (automatic titration group only), duration of hospital admission, number of medical emergency team (MET) calls and requirement for noninvasive ventilation or intensive care unit (ICU) admission.

\section{Sample size}

Based on two previous studies investigating a closed-loop control oxygen delivery system, ${ }^{12}{ }^{13}$ a total sample size of 46 participants was estimated to provide $90 \%$ power to detect a $30 \%$ difference in time spent in target range between intervention and control based on an SD of 30. The sample size calculations were based on using an unpaired t-test to compare groups, equal size numbers in intervention and control groups, and a two-sided type I error rate (alpha) of 5\%. Due to uncertainty whether this sample size calculation was applicable to the performance of the NHF device, a prespecified sample size re-estimation was performed after 10 participants which indicated a revised total of 20 participants was required to provide at least $90 \%$ power to detect a $30 \%$ difference in time spent within target $\mathrm{SpO}_{2}$ range. A minimum of 6 hours data was required for the participant to be included in the analysis. Additional participants were recruited to meet the sample size if less than 6 hours of data was collected. As a result, a per protocol analysis was undertaken in those participants in whom at least 6 hours of data was available.

\section{Statistical analysis}

The primary analysis was pre-specified as a general linear model (analysis of covariance, ANCOVA), however, due to a skewed data distribution, a Wilcoxon rank-based method with the Hodges-Lehmann estimator for location difference and appropriate confidence intervals was used for all outcomes relating to $\mathrm{SpO}_{2}$. Other outcomes

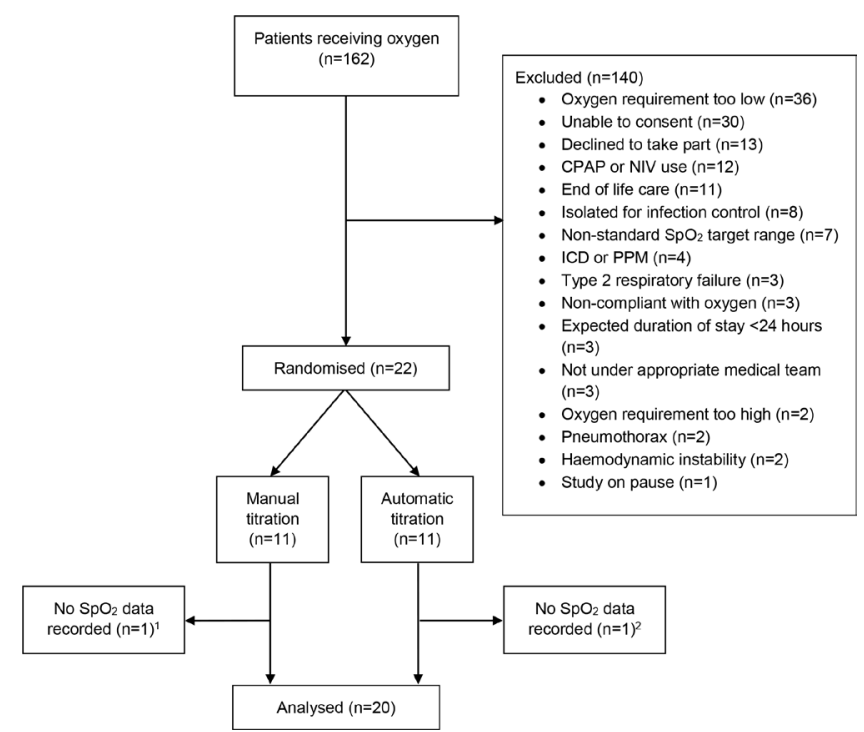

Figure 1 Consort flow diagram. ${ }^{1}$ A technical fault caused no $\mathrm{SpO}_{2}$ data to be recorded. ${ }^{2} \mathrm{SpO}_{2}$ was found to be within range while breathing room air and the participant was withdrawn prior to starting the randomised treatment, as a result, no $\mathrm{SpO}_{2}$ data were recorded. CPAP, continuous positive airway pressure; ICD, implanted cardioverter defibrillator; NIV, non-invasive ventilation; PPM, permanent pacemaker; $\mathrm{SpO}_{2}$, oxygen saturation.

were analysed using ANCOVA with adjustment for baseline or by t-test where no baseline was appropriate. Mean (SD) values are presented for normally distributed variables and median (IQR) for other variables. SAS V.9.4 (SAS Institute) was used.

\section{RESULTS}

Participants were recruited to the trial between 9 January 2020 and 2 October $2020 .^{24}$ A total of 162 patients receiving oxygen were screened and 22 were randomised. Twenty participants with greater than 6 hours of recorded data were included in the per-protocol analysis. The overall mean (SD) duration of intervention for participants included in the analysis was 23.2 (2.1) hours and 22.4 (3.8) hours in the manual and automatic titration groups, respectively. One participant in the automatic titration group was withdrawn from the study after 12.3 hours due to an increase in oxygen requirement in excess of the protocol defined limit. There was a device fault for a different participant in the automatic titration group which resulted in an unexpected shut down of closed-loop control after 19.7 hours. Two participants in the manual titration group finished the intervention period after 17.5 hours and 22.5 hours, respectively, as the treating clinical team wished to stop oxygen therapy. The trial profile is shown in figure 1. Baseline participant characteristics are shown in table 1. Baseline and postintervention capillary blood gas values according to prescribed target range are shown in table 2. Participant characteristics and blood gas values were similar between treatment groups. The mean (SD) percentage of time 


\begin{tabular}{|c|c|c|}
\hline Characteristic & $\begin{array}{l}\text { NHF manual } \\
\mathrm{N}=10\end{array}$ & $\begin{array}{l}\text { NHF automatic } \\
\mathrm{N}=10\end{array}$ \\
\hline \multicolumn{3}{|l|}{ Sex } \\
\hline Female & 4 & 4 \\
\hline \multicolumn{3}{|l|}{ Ethnicity } \\
\hline European & 9 & 10 \\
\hline Pacific peoples & 1 & 0 \\
\hline \multicolumn{3}{|l|}{ Reason for oxygen } \\
\hline $\begin{array}{l}\text { Exacerbation of } \\
\text { asthma }\end{array}$ & 2 & 1 \\
\hline $\begin{array}{l}\text { Exacerbation of } \\
\text { COPD }\end{array}$ & 3 & 3 \\
\hline Heart failure & 1 & 1 \\
\hline $\begin{array}{l}\text { Interstitial lung } \\
\text { disease }\end{array}$ & 1 & 1 \\
\hline Pulmonary embolism & 1 & 0 \\
\hline Pneumonia & 2 & 4 \\
\hline \multicolumn{3}{|l|}{ Target $\mathrm{SpO}_{2}$ range } \\
\hline $88 \%-92 \%$ & 5 & 6 \\
\hline $92 \%-96 \%$ & 5 & 4 \\
\hline \multicolumn{3}{|l|}{ High-dependency area } \\
\hline Yes & 5 & 4 \\
\hline \multicolumn{3}{|c|}{ Mean (SD) } \\
\hline Age (years) & $69.2(19)$ & $68.7(17)$ \\
\hline Heart rate (beats/min) & $80.2(17.2)$ & $89.9(15.1)$ \\
\hline Respiratory rate & 21.7 (3.3) & $23.4(5.9)$ \\
\hline Systolic blood pressure & $130(20)$ & $118.1(16.1)$ \\
\hline Diastolic blood pressure & $70.4(9)$ & $72.3(10.7)$ \\
\hline Baseline $\mathrm{FiO}_{2}(\%)$ & $30.6(3.5)$ & $28.3(2)$ \\
\hline
\end{tabular}

COPD, chronic obstructive pulmonary disease; $\mathrm{FiO}_{2}$, fractional inspired oxygen; $\mathrm{SpO}_{2}$, oxygen saturation.

with adequate signal quality was high and similar between the two interventions; 96.1 (2.9)\% and $91.3(5.8) \%$ in the automatic and manual titration groups, respectively.

For the primary outcome, the automatic titration group spent a median (IQR) of 96.2\% (95.2-97.8) of the intervention period with $\mathrm{SpO}_{2}$ within target range, compared with $71 \%$ (59.4-88.3) in the manual titration group. The difference (95\% CI) between the groups was $24.2 \%$ ( $7.9 \%$ to $35 \%), \mathrm{p}<0.001$; table 3 and figure 2 . This difference was $24.3 \%$ (11.7 to 37.6), $\mathrm{p}<0.001$ when all $\mathrm{SpO}_{2}$ data, irrespective of signal quality was used for analysis.

For the secondary outcomes, there was a trend towards a reduction in time spent with $\mathrm{SpO}_{2} \geq 2 \%$ below range in the automatic titration group; difference $-2.4 \%(-11.5$ to 0.3$), p=0.05$ and a significant reduction in time spent with $\mathrm{SpO}_{2} \geq 2 \%$ above target range; difference $-1 \%(-8.2$ to -0.04$), p=0.017$. The maximum delivered oxygen concentration during the intervention period was significantly higher in the automatic titration group; mean difference adjusted for baseline (95\% CI) $7.1 \%$ (4.6\% to $9.7 \%), \mathrm{p}<0.001$. There was no difference in blood gas measurements or in the other secondary outcomes between interventions. Primary and secondary outcomes are summarised in table 3 and online supplemental table S1. Histogram density plots for individual participants according to randomised treatment and target $\mathrm{SpO}_{2}$ range are shown in figures 3 and 4 .

For the tertiary outcomes, the mean (SD) duration of hospital admission was 4.8 (2.6) days in the manual titration group and 8.1 (6) days in the automatic titration group. In the automatic titration group the median (IQR) percentage of time spent with $\mathrm{SpO}_{2}$ below target range and $\mathrm{FiO}_{2}$ at the upper limit was $1.1 \%(0.6-2.5)$ and with $\mathrm{SpO}_{2}$ above range and $\mathrm{FiO}_{2}$ at the lower limit was $0.2 \%(0-1.3)$.

Two post hoc analyses were performed. First, a subgroup analysis for the primary outcome according to target $\mathrm{SpO}_{2}$ range demonstrated a greater proportion of time spent in target range for the automatic titration group in both the 88\%-92\% target range and the 92\%-96\% target range; difference $28.1 \%$ (6.9-45.4), $\mathrm{p}=0.018 \%$ and $23.3 \%$ ( -10.3 to 5.2$), \mathrm{p}=0.028$ respectively. Asymptotic assumptions were not met for the small samples hence confidence intervals do not correspond well to $\mathrm{p}$ values. Second, an analysis of proportion of time spent in target range during day and night (23:00-07:00 hour) demonstrated consistent performance of automatic titration with median (IQR) 96.6\% (93.9-98.5) and 96.3\% (93.6-98.1), respectively. In the manual titration group a higher proportion of time was spent in range during the day compared with overnight $77.5 \%(65.3-85.1)$ and $66.6 \%$ (46.4-85.4), respectively.

There was one MET call, one ICU admission and one death involving the same participant in the automatic titration group. This was considered to be a serious adverse event which was reviewed by the DSMC, and was determined to be unrelated to the study intervention. The device fault which occurred in a different participant in the automatic titration group, causing an unexpected shut down of closed-loop control, was also reviewed by the DSMC. This did not lead to any adverse effects for the participant and the fault was remedied by a software update prior to further participant recruitment.

\section{DISCUSSION}

This trial demonstrates medical inpatients with an acute illness spend $96 \%$ of time with $\mathrm{SpO}_{2}$ within a prescribed target range while receiving oxygen delivered using NHF with automatic oxygen titration. This is significantly higher than the $71 \%$ of time spent within target range using NHF with manual oxygen titration, which is current standard practice. Over the 24-hour intervention period, this equates to approximately six additional hours spent with $\mathrm{SpO}_{2}$ within target range, which may be of clinical importance.

The reduction in time spent with $\mathrm{SpO}_{2} \geq 2 \%$ above and below target range with automatic titration suggests that 
Table 2 Baseline and postintervention capillary blood gas results

\begin{tabular}{|c|c|c|c|c|c|c|}
\hline \multirow[b]{2}{*}{ Target $\mathrm{SpO}_{2}$ range } & \multicolumn{3}{|c|}{ NHF manual } & \multicolumn{3}{|c|}{ NHF automatic } \\
\hline & $\begin{array}{l}88 \%-92 \% \\
\mathrm{~N}=5\end{array}$ & $\begin{array}{l}92 \%-96 \% \\
\mathrm{~N}=5\end{array}$ & $\begin{array}{l}\text { All } \\
\mathrm{N}=10\end{array}$ & $\begin{array}{l}88 \%-92 \% \\
\mathrm{~N}=6\end{array}$ & $\begin{array}{l}92 \%-96 \% \\
\mathrm{~N}=4\end{array}$ & $\begin{array}{l}\text { All } \\
\mathrm{N}=10\end{array}$ \\
\hline Baseline & Mean (SD) & & & & & \\
\hline $\mathrm{pH}$ & $7.44(0.02)$ & $7.47(0.06)$ & $7.5(0)$ & $7.46(0.06)$ & $7.47(0.02)$ & $7.5(0)$ \\
\hline $\mathrm{PCO}_{2}(\mathrm{~mm} \mathrm{Hg})$ & $35.9(6.2)$ & $30.5(4.9)$ & $33.2(6)$ & $40.0(7.0)$ & $32.6(5.2)$ & $37(7.1)$ \\
\hline $\mathrm{PO}_{2}(\mathrm{~mm} \mathrm{Hg})$ & $59.0(4.7)$ & $68.9(9.3)$ & $63.9(8.7)$ & $62.6(12.9)$ & $68.9(6.9)$ & $65.1(10.9)$ \\
\hline Target $\mathrm{SpO}_{2}$ range & $\begin{array}{l}88 \%-92 \% \\
\mathrm{~N}=5\end{array}$ & $\begin{array}{l}92 \%-96 \% \\
\mathrm{~N}=5\end{array}$ & $\begin{array}{l}\text { All } \\
\mathrm{N}=10\end{array}$ & $\begin{array}{l}88 \%-92 \% \\
\mathrm{~N}=6\end{array}$ & $\begin{array}{l}92 \%-96 \% * \\
\mathrm{~N}=3\end{array}$ & $\begin{array}{l}\text { All } \\
\mathrm{N}=9\end{array}$ \\
\hline Postintervention & Mean (SD) & & & & & \\
\hline $\mathrm{pH}$ & $7.46(0.02)$ & $7.47(0.05)$ & $7.5(0)$ & $7.47(0.04)$ & $7.47(0.02)$ & $7.5(0)$ \\
\hline $\mathrm{PCO}_{2}(\mathrm{~mm} \mathrm{Hg})$ & $35.9(6.1)$ & $30.7(6.2)$ & $33.3(6.4)$ & $40.4(6.5)$ & $34.0(4.7)$ & $38.2(6.5)$ \\
\hline $\mathrm{PO}_{2}(\mathrm{~mm} \mathrm{Hg})$ & $56.6(5.6)$ & $73.4(12.6)$ & 65 (12.8) & $56.1(6.0)$ & $74.2(8.7)$ & $62.1(11.1)$ \\
\hline
\end{tabular}

${ }^{*}$ One participant in the automatic titration 92\%-96\% group was withdrawn and subsequently required mechanical ventilation, hence an end of intervention blood gas was not taken.

$\mathrm{NHF}$, nasal high-flow; $\mathrm{PCO}_{2}$, partial pressure of carbon dioxide; $\mathrm{PO}_{2}$, partial pressure of oxygen.

this novel device has the potential to reduce the risks associated with both hypoxaemia and hyperoxaemia. Although the point estimates for this difference were small, there was large variability in the manual titration group, illustrated by the wide IQR for these outcomes and correspondingly wide CIs for the difference. In comparison, there was a consistent reduction in time spent outside of range in the automatic titration group, well illustrated in the histogram density plots in figures 3 and 4 where a tall narrow distribution around the target range was observed for automatic titration and a more variable wider distribution for manual titration. The greater proportion of time spent with $\mathrm{SpO}_{2} \geq 2 \%$ above compared with below range in the manual titration group suggests that healthcare professionals aim for a margin of comfort with the delivery of oxygen therapy, even with a specified target $\mathrm{SpO}_{2}$ range. The mean number of manual $\mathrm{FiO}_{2}$ adjustments in the manual titration group was comparable to the number of $\mathrm{FiO}_{2}$ range adjustments performed by nursing staff in the automatic titration group, suggesting that for a similar degree of nursing staff input, a significantly greater proportion of time with $\mathrm{SpO}_{2}$ in target range can be achieved. Although not evaluated in this study, automatic oxygen titration may reduce the nursing

Table 3 Outcomes

\begin{tabular}{|c|c|c|c|c|}
\hline \multirow[b]{2}{*}{ Outcome } & NHF manual $\mathrm{N}=10$ & NHF automatic $\mathbf{N}=10$ & \multirow{2}{*}{$\begin{array}{l}\text { Difference }(95 \% \mathrm{Cl}) \\
\text { Rank based }\end{array}$} & \multirow[b]{2}{*}{$P$ value } \\
\hline & Median (IQR) & & & \\
\hline Time in target $\mathrm{SpO}_{2}$ range (\%) & 71 (59.4 to 88.3$)$ & $96.2(95.2$ to 97.8$)$ & 24.2 (7.9 to 35$)$ & $<0.001$ \\
\hline Time in target $\mathrm{SpO}_{2}$ range-all $\mathrm{SQ}(\%)$ & 69 (52 to 82.4$)$ & 93.3 (89.7 to 97.1$)$ & 24.3 (11.7 to 37.6$)$ & $<0.001$ \\
\hline Time $\geq 2 \%$ above range $(\%)$ & $5.6(9)$ & $0.8(2.2)$ & $-1(-8.2$ to -0.04$)$ & 0.017 \\
\hline Time $\geq 4 \%$ above range $(\%)$ & $0(0$ to 0.4$)$ & 0 (0 to 0$)$ & $-0.01(-0.25$ to 0$)$ & 0.021 \\
\hline Time $\geq 2 \%$ below range $(\%)$ & $1.5(0.5$ to 8.2$)$ & $0.1(0$ to 0.2$)$ & $-2.4(-11.5$ to 0.3$)$ & 0.05 \\
\hline Time $\geq 4 \%$ below range $(\%)$ & 0 (0 to 0.3$)$ & $0(0$ to 0$)$ & $-0.25(-4.8$ to 0.4$)$ & 0.31 \\
\hline \multirow[t]{2}{*}{ Time $\geq 8 \%$ below range $(\%)$} & 0.1 (0 to 1.6$)$ & 0.1 (0 to 0.2$)$ & $-0.01(-1.5$ to 0.1$)$ & 0.54 \\
\hline & Mean (SD) & & Mean difference* & \\
\hline Mean heart rate (beats/min) & $78(13.1)$ & $87.9(15.1)$ & $0.5(-8.2$ to 9.1$)$ & 0.91 \\
\hline Mean $\mathrm{FiO}_{2}(\%)$ & $29.4(3.5)$ & $29.1(2)$ & $1.5(-0.5$ to 3.4$)$ & 0.13 \\
\hline Minimum $\mathrm{FiO}_{2}(\%)$ & $25.3(4.2)$ & $23.3(2.6)$ & $-0.5(-3.5$ to 2.6$)$ & 0.76 \\
\hline Maximum $\mathrm{FiO}_{2}(\%)$ & $32(4.3)$ & $37.1(2.7)$ & 7.1 (4.6 to 9.7$)$ & $<0.001$ \\
\hline No of manual $\mathrm{FiO}_{2}$ changes $\dagger$ & $4.8(5.4)$ & $4(3.9)$ & $-0.8(-5.3$ to 3.7$)$ & 0.35 \\
\hline No of automatic $\mathrm{FiO}_{2}$ changes & $\mathrm{N} / \mathrm{A}$ & $72103(15118)$ & $\mathrm{N} / \mathrm{A}$ & $\mathrm{N} / \mathrm{A}$ \\
\hline
\end{tabular}

${ }^{*}$ Adjusted for baseline, other than number of manual $\mathrm{FiO}_{2}$ changes.

†In the automatic group the number of manual $\mathrm{FiO}_{2}$ changes refers to the number of times the $\mathrm{FiO}_{2}$ limits were changed by nursing staff. $\mathrm{FiO}_{2}$, fractional inspired oxygen; NA, not available; NHF, nasal high-flow. 


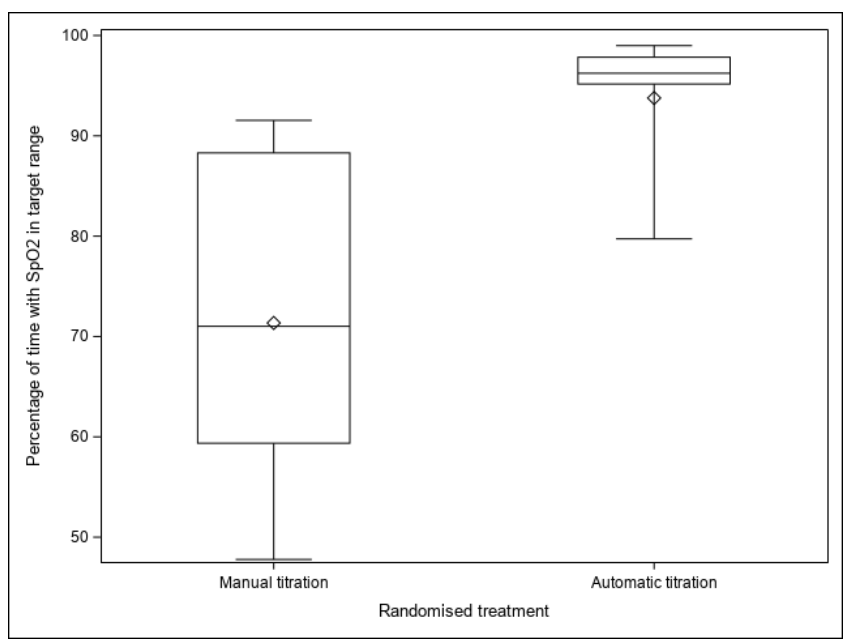

Figure 2 Box plot of proportion of time spent with $\mathrm{SpO}_{2}$ in target range according to randomised treatment. On the box-plots the symbol is the mean, the horizontal lines are the 25th, 50th (median) and 75th percentiles, and the whiskers extend from the minimum to maximum values. $\mathrm{SpO}_{2}$, oxygen saturation.

time spent at the bedside titrating oxygen, freeing nursing resources for other aspects of clinical care.

Compared with our results, previous studies of conventional oxygen therapy with automatic titration have demonstrated a lower mean $81.2 \%$ and $81.3 \%$ proportion of time spent with $\mathrm{SpO}_{2}$ in target range in patients admitted to hospital with an acute exacerbation of $\mathrm{COPD}^{13}$ and hypoxaemic patients in the $\mathrm{ED},{ }^{12}$ respectively. The manual titration group of these studies also spent a lower proportion of time in range, $51.3(19.7) \%^{13}$ and $51.8(30) \%^{12}$ as compared with the manual titration group in this study. This may be due to the relatively high proportion of participants who were in a high-dependency area where continuous oximetry is typically used, differing participant characteristics or the

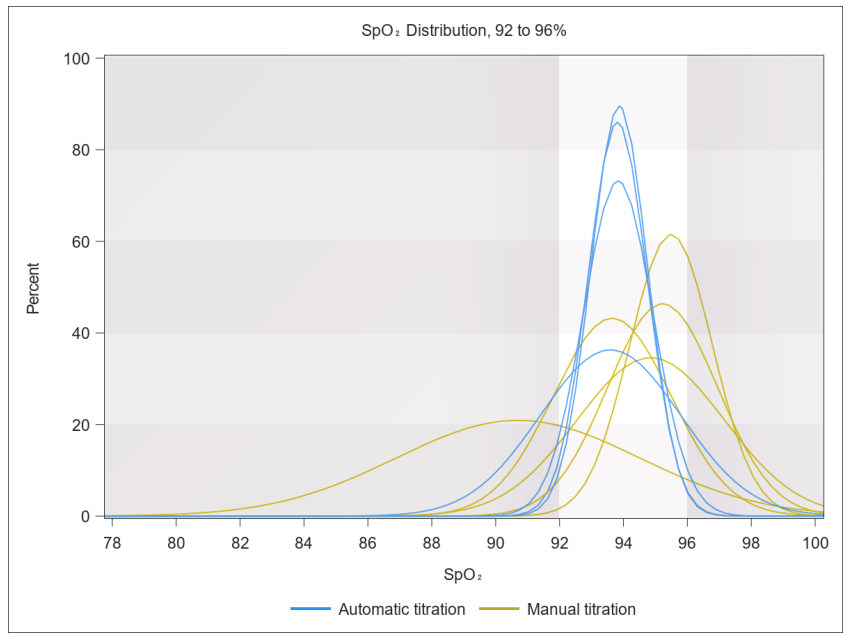

Figure 3 Histogram density plot of $\mathrm{SpO}_{2}$ measurements during the intervention period for individual participants with a target range of $92 \%-96 \%$. The lightly shaded area respresents the target range. $\mathrm{SpO}_{2}$, oxygen saturation.

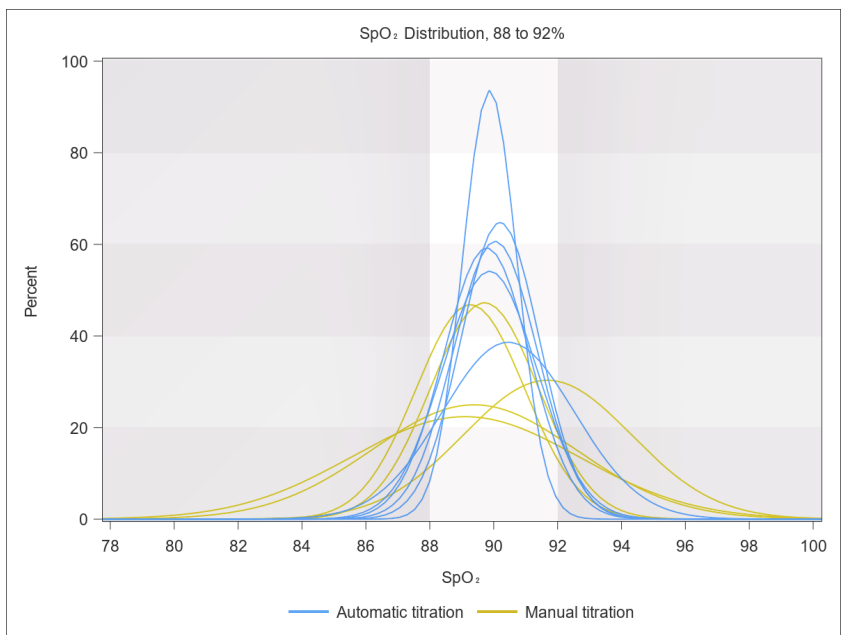

Figure 4 Histogram density plot of $\mathrm{SpO}_{2}$ measurements during the intervention period for individual participants with a target range of $88 \%-92 \%$. The lightly shaded area respresents the target range. $\mathrm{SpO}_{2}$, oxygen saturation.

presence of a bedside guide indicating target $\mathrm{SpO}_{2}$ range and prompting intervention if $\mathrm{SpO}_{2}$ was out of range in this study.

The strengths of this study include the use of NHF in both groups, ensuring any differences are due to the mechanism of oxygen titration. In addition, oxygen was titrated at the start of the intervention period to ensure all participants were within target range ensuring a fair starting point. However, there are a number of considerations when interpreting the results. First, while the study was adequately powered for the primary outcome variable, it was underpowered to detect a difference between interventions in terms of significant time exposure to marked over or under-oxygenation. The study was also not powered to detect differences in clinical outcomes. Second, the participants included had mild to moderate hypoxaemia and so are not generalisable to a population with greater oxygen requirements or who are more unstable. Patients requiring more than $40 \%$ oxygen were excluded from this study, a group likely to benefit from NHF therapy, ${ }^{22}$ however, this exclusion criterion was used to enhance safety as this study was the first time this novel method of oxygen delivery had been used in an inpatient setting. Lastly, the continuous display of $\mathrm{SpO}_{2}$ and use of alarms in the automatic titration group may have alerted nursing staff to deviation from the target range more rapidly than in the manual titration group, where continuous oximetry was used at the discretion of the nursing and medical team. These factors are, however, an intrinsic part of the automatic titration system and can be viewed as an advantage over standard care. The potential issue of 'alarm fatigue' will need to be addressed in future studies over a longer duration of intervention.

In conclusion, this study provides evidence that NHF with automatic oxygen titration using the Airvo 3 device results in a greater proportion of time spent with $\mathrm{SpO}_{2}$ within target range compared with NHF with manual 
oxygen titration in medical patients with hypoxaemia. These results need confirmation in larger studies and in different patient populations and clinical settings. Further investigation as to whether the use of nasal high flow with automatic oxygen titration influences clinical outcomes such as escalation of care and hospital length of stay is also warranted.

Acknowledgements We thank all participants who contributed to this study, taking part in a research study at a time of acute illness is a burden which we greatly appreciate. We also thank the nursing staff of wards 5 South, 6 South and MAPU in Wellington Hospital for their assistance with the study.

Contributors $\mathrm{JH}, \mathrm{RB}$ and IB designed the study. $\mathrm{JH}$ was principal investigator and NK and GB were investigators. NS designed and maintained the electronic study database. AE and MW performed the data processing and statistical analysis. $\mathrm{JH}$ wrote the first draft of the manuscript and all authors contributed to the final version.

Funding This study was funded by Fisher and Paykel Healthcare, Auckland, New Zealand.

Disclaimer The funder had no role in the conduct of the study, data analysis or writing of the manuscript.

Competing interests $\mathrm{JH}$ reports personal fees from Fisher and Paykel Healthcare, outside the submitted work. RB reports a grant from Fisher and Paykel Healthcare to undertake this study.

Patient consent for publication Not required.

Ethics approval The trial was approved by the Northern A Health and Disability Ethics Committee.

Provenance and peer review Not commissioned; externally peer reviewed.

Data availability statement Data are available on reasonable request. Individual participant data that underlie the results reported in this article, after deidentification(text, tables, figures and appendices) will be avaliable one year after publication until a minimum of 5 years after publication to researchers who provide a methodologically sound proposal that has been approved by the study steering committee and sponsor to achieve the aims outlined in the approved proposal. This is possible through a signed data access agreement and subject to approval by the principal investigator (james.harper@mrinz.ac.nz) and the study sponsor (james. revie@fphcare.co.nz). No other documents will be available.

Supplemental material This content has been supplied by the author(s). It has not been vetted by BMJ Publishing Group Limited (BMJ) and may not have been peer-reviewed. Any opinions or recommendations discussed are solely those of the author(s) and are not endorsed by BMJ. BMJ disclaims all liability and responsibility arising from any reliance placed on the content. Where the content includes any translated material, BMJ does not warrant the accuracy and reliability of the translations (including but not limited to local regulations, clinical guidelines, terminology, drug names and drug dosages), and is not responsible for any error and/or omissions arising from translation and adaptation or otherwise.

Open access This is an open access article distributed in accordance with the Creative Commons Attribution Non Commercial (CC BY-NC 4.0) license, which permits others to distribute, remix, adapt, build upon this work non-commercially, and license their derivative works on different terms, provided the original work is properly cited, appropriate credit is given, any changes made indicated, and the use is non-commercial. See: http://creativecommons.org/licenses/by-nc/4.0/.

ORCID iDs

James Harper http://orcid.org/0000-0002-6416-1466

Irene Braithwaite http://orcid.org/0000-0001-5327-3027

\section{REFERENCES}

1 O'Driscoll BR, Howard LS, Bucknall C, et al. British thoracic Society emergency oxygen audits. Thorax 2011;66:734-5.

2 O'Driscoll BR, Howard LS, Earis J, et al. BTS guideline for oxygen use in adults in healthcare and emergency settings. Thorax 2017;72:ii1-90.

3 Beasley R, Chien J, Douglas J, et al. Thoracic Society of Australia and New Zealand oxygen guidelines for acute oxygen use in adults: 'Swimming between the flags'. Respirology 2015;20:1182-91.
4 Beasley R, Mackle D, Young P. Oxygen: a new look at an old therapy $J$ R Soc N Z 2019;49:126-42.

5 Buist M, Bernard S, Nguyen TV, et al. Association between clinically abnormal observations and subsequent in-hospital mortality: a prospective study. Resuscitation 2004;62:137-41.

6 Chu DK, Kim LH-Y, Young PJ, et al. Mortality and morbidity in acutely ill adults treated with liberal versus conservative oxygen therapy (IOTA): a systematic review and meta-analysis. Lancet 2018;391:1693-705.

7 Siemieniuk RAC, Chu DK, Kim LH-Y, et al. Oxygen therapy for acutely ill medical patients: a clinical practice guideline. BMJ 2018;363:k4169-10.

8 Barrot L, Asfar P, Mauny F, et al. Liberal or conservative oxygen therapy for acute respiratory distress syndrome. N Engl J Med 2020;382:999-1008.

9 ICU-ROX investigators and the Australian and New Zealand intensive care society clinical trials group, Mackle D, Bellomo R, et al. Conservative oxygen therapy during mechanical ventilation in the ICU. N Engl J Med 2020;382:989-98.

10 British thoracic Society emergency oxygen audit report national audit period: 15 August - 1 November 2015, 2015. Available: https:// www.brit-thoracic.org.uk/document-library/quality-improvement/ audit-reports/emergency-oxygen-2015/

11 L'Her E, Jaber S, Verzilli D, et al. Automated closed-loop versus standard manual oxygen administration after major abdominal or thoracic surgery: an international multicentre randomised controlled study. Eur Respir J.

12 L'Her E, Dias P, Gouillou M, et al. Automatic versus manual oxygen administration in the emergency department. Eur Respir J 2017;50. doi:10.1183/13993003.02552-2016. [Epub ahead of print: 20 Jul 2017].

13 Lellouche F, Bouchard P-A, Roberge M, et al. Automated oxygen titration and weaning with FreeO2 in patients with acute exacerbation of COPD: a pilot randomized trial. Int J Chron Obstruct Pulmon Dis 2016:11:1983-90.

14 Malli F, Boutlas S, Lioufas N, et al. Automated oxygen delivery in hospitalized patients with acute respiratory failure: a pilot study. Can Respir J 2019;2019:1-7.

15 Hansen EF, Hove JD, Bech CS, et al. Automated oxygen control with O2matic ${ }^{\circledR}$ during admission with exacerbation of COPD. Int $J$ Chron Obstruct Pulmon Dis 2018;13:3997-4003.

16 Parke RL, McGuinness SP. Pressures delivered by nasal high flow oxygen during all phases of the respiratory cycle. Respir Care 2013;58:1621-4.

17 Mauri T, Turrini C, Eronia N, et al. Physiologic effects of high-flow nasal cannula in acute hypoxemic respiratory failure. Am J Respir Crit Care Med 2017;195:1207-15.

18 Ritchie JE, Williams AB, Gerard C, et al. Evaluation of a humidified nasal high-flow oxygen system, using oxygraphy, capnography and measurement of upper airway pressures. Anaesth Intensive Care 2011;39:1103-10.

19 Wagstaff TAJ, Soni N. Performance of six types of oxygen delivery devices at varying respiratory rates. Anaesthesia 2007:62:492-503.

20 Möller W, Feng S, Domanski U, et al. Nasal high flow reduces dead space. J Appl Physiol 2017;122:191-7.

21 Ferreyro BL, Angriman F, Munshi L, et al. Association of noninvasive oxygenation strategies with all-cause mortality in adults with acute hypoxemic respiratory failure: a systematic review and metaanalysis. JAMA 2020;324:57-67.

22 Rochwerg B, Granton D, Wang DX, et al. High flow nasal cannula compared with conventional oxygen therapy for acute hypoxemic respiratory failure: a systematic review and meta-analysis. Intensive Care Med 2019;45:563-72.

23 Ischaki E, Pantazopoulos I, Zakynthinos S. Nasal high flow therapy: a novel treatment rather than a more expensive oxygen device. Eur Respir Rev 2017;26. doi:10.1183/16000617.0028-2017. [Epub ahead of print: 30 Sep 2017].

24 Harper JC, Kearns NA, Maijers I, et al. Closed-Loop oxygen control using a novel nasal high-flow device: a randomized crossover trial. Respir Care 2021;66:416-24. doi:10.4187/respcare.08087

25 Harris PA, Taylor R, Minor BL, et al. The REDCap Consortium: building an international community of software platform partners. $J$ Biomed Inform 2019;95:103208.

26 Harris PA, Taylor R, Thielke R, et al. Research electronic data capture (REDCap)--a metadata-driven methodology and workflow process for providing translational research informatics support. J Biomed Inform 2009:42:377-81. 